\title{
Single crystals of electrically conductive 2D MOFs: structural and electrical transport properties
}

Robert W. Day ${ }^{1}$, D. Kwabena Bediako ${ }^{2}$, Mehdi Rezaee ${ }^{3}$, Lucas R. Parent ${ }^{4,5}$,

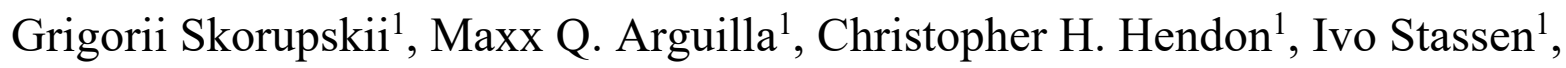
Nathan C. Gianneschi ${ }^{4}$, Philip Kim² ${ }^{2}$, and Mircea Dincă ${ }^{1 *}$

${ }^{1}$ Department of Chemistry, Massachusetts Institute of Technology, Cambridge, Massachusetts 02138, USA.

${ }^{2}$ Department of Physics, Harvard University, Cambridge, Massachusetts 02138, USA.

${ }^{3}$ John A. Paulson School of Engineering and Applied Sciences, Harvard University, Cambridge, Massachusetts 02138, USA.

${ }^{4}$ Department of Chemistry, Materials Science \& Engineering, Biomedical Engineering, International Institute for Nanotechnology, Northwestern University, Evanston, Illinois 60208, USA.

${ }^{5}$ Innovation Partnership Building, University of Connecticut, Storrs, CT 06269, USA.

*email:mdinca@mit.edu 
Crystalline, electrically conductive, and intrinsically porous materials are rare. Layered 2D metal-organic frameworks (MOFs) break this trend. They are porous crystals that exhibit high electrical conductivity and are novel platforms for studying fundamentals of electricity and magnetism in two dimensions. ${ }^{1-8}$ Despite demonstrated applications, ${ }^{9-13}$ electrical transport in these remains poorly understood because of a lack of single crystal studies. Here, studies of single crystals of two 2D MOFs, $\mathrm{Ni}_{3}(\mathrm{HITP})_{2}$ and $\mathrm{Cu}_{3}(\mathrm{HHTP})_{2}$, uncover critical insights into their structure and transport. Conductivity measurements down to $0.3 \mathrm{~K}$ suggest metallicity for mesoscopic single crystals of $\mathrm{Ni}_{3}(\mathrm{HITP})_{2}$, which contrasts with apparent activated conductivity for polycrystalline films. Microscopy studies further reveal that these MOFs are not isostructural as previously reported. ${ }^{14}$ Notably, single rods exhibit conductivities up to $150 \mathrm{~S} / \mathrm{cm}$, which persist even after prolonged exposure to the ambient. These single crystal studies confirm that 2D MOFs hold promise as molecularly tunable platforms for fundamental science and applications where porosity and conductivity are critical.

MOFs are hybrid inorganic/organic crystals that can possess features distinct from many crystalline materials: ${ }^{15,16}$ intrinsic porosity, nanometer-scale lattice parameters, flexible mechanics, and a rich structural and compositional diversity enabled by advances in both organic and inorganic chemistry in three dimensions. The vast majority of MOFs are insulators and have been used in applications that benefit from high surface areas and a chemical tunability, such as gas capture and catalysis. ${ }^{15}$ Conductive MOFs represent a new type of hybrid inorganic/organic conductor in addition to non-porous coordination polymers and hybrid perovskites, which have recently demonstrated high conductivities and superconductivity ${ }^{17}$ and promising performances for optoelectronics, ${ }^{18}$ respectively.

Two-dimensional (2D) layered MOFs where metals and redox-active ligands form extended $\pi$-d conjugated sheets (Fig. 1), have shown the highest conductivities and are predicted to exhibit rare transport phenomena including, for instance, the quantum anomalous hall effect. ${ }^{7}$ However, due to poor synthetic control and small crystallite size, charge transport and structural studies for the 2D MOFs have generally relied on polycrystalline films/pellets and powder X-ray diffraction (PXRD), where grain boundaries and anisotropy obscure the intrinsic properties. Only a handful of single crystal devices have been reported including a room-temperature conductivity 
value for a $2 \mathrm{D} \mathrm{MOF},{ }^{19}$ for a $2 \mathrm{D}$ coordination polymer, ${ }^{20}$ and for some less-conductive $3 \mathrm{D}$ $\mathrm{MOFs}^{21,22}$. Despite $\mathrm{Ni}_{3}(2,3,6,7,10,11 \text {-hexaiminotriphenylene })_{2}\left(\mathrm{Ni}_{3}(\mathrm{HITP})_{2}\right)$ possessing one of the highest conductivities for a porous MOF and having demonstrated promise for various applications, its conduction is poorly understood; calculations have predicted $\mathrm{Ni}_{3}(\mathrm{HITP})_{2}$ to be metallic but results of experiments on polycrystalline pellets down to $80 \mathrm{~K}$ have been interpreted as semiconducting. ${ }^{4,23}$ Here, with isolated crystals, we reveal the distinct layer stacking for $\mathrm{Ni}_{3}(\mathrm{HITP})_{2}$ and $\mathrm{Cu}_{3}(2,3,6,7,10,11 \text {-hexahydroxytriphenylene })_{2}\left(\mathrm{Cu}_{3}(\mathrm{HHTP})_{2}\right)$, the metallic nature of single crystals of $\mathrm{Ni}_{3}(\mathrm{HITP})_{2}$, and the importance of transport in the out-of-plane direction despite the presumed dominance of in-plane transport for 2D MOFs.

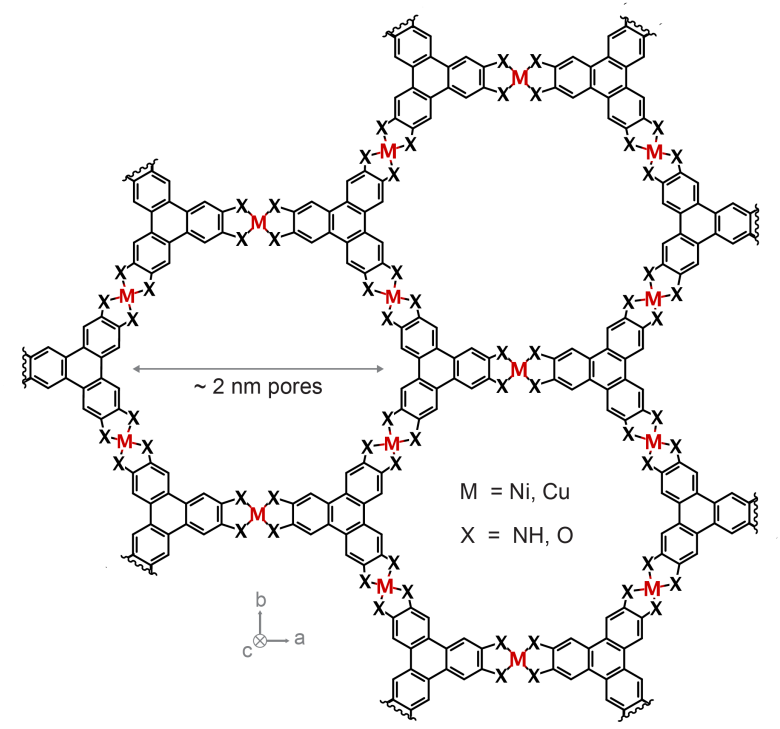

Figure 1 | Porous, conductive 2D MOFs. Schematic illustrating the general structure of conductive 2D MOFs where layers stack in the $c$ direction to form intrinsically porous crystals with pores of approximately $2 \mathrm{~nm}$ in diameter. Metal atoms and organic ligands comprise the honeycomb lattice of the $2 \mathrm{D}$ layers. $\mathrm{Ni}_{3}(\mathrm{HITP})_{2}$ and $\mathrm{Cu}_{3}(\mathrm{HHTP})_{2}$ have $\mathrm{M} / \mathrm{X}=\mathrm{Ni} / \mathrm{NH}$ and $\mathrm{Cu} / \mathrm{O}$ respectively.

To understand charge transport in our 2D MOFs, we isolated individual crystals of $\mathrm{Ni}_{3}$ (HITP) $)_{2}$, and measured their conductivity as a function of temperature. (Fig. 2) Published conditions $^{4}$ generate an interconnected nanocrystalline morphology (Supplementary Figure 1), which has been challenging to isolate crystals from. Here, we obtain isolated rods of $\mathrm{Ni}_{3}(\mathrm{HITP})_{2}$ up to $\sim 2 \mu \mathrm{m}$ in length and $\sim 200 \mathrm{~nm}$ in diameter (see Methods and Supplementary Figure 1). 
Devices were fabricated by drop-casting suspensions of $\mathrm{Ni}_{3}(\mathrm{HITP})_{2}$ rods onto $\mathrm{Si}$ covered by $\mathrm{SiO}_{2}$ substrates and using electron-beam lithography and metal evaporation to deposit $\mathrm{Ti} / \mathrm{Pd}$ contacts on top of the rods (Fig. 2a; see Methods). A DC current-voltage (I-V) sweep for a representative device (Fig. 2b) yielded four-probe conductance values $G$ of $1.3 \mathrm{mS}$ at $295 \mathrm{~K}$ and $0.7 \mathrm{mS}$ at 1.4 $\mathrm{K}$, and two-probe values of $0.25 \mathrm{mS}$ at $295 \mathrm{~K}$ and $0.13 \mathrm{mS}$ at $1.4 \mathrm{~K}$. The linearity observed in these DC measurements even at small biases evinces ohmic contacts over this temperature range and validates the use of $\mathrm{AC}$ transport measurements for variable temperature (VT) and magnetic field-dependence studies. A reversible decrease in conductance is observed when a magnetic field is applied (Fig. 2b, inset), and the magnitude of this change decreases as the temperature $T$ increases from $1.4 \mathrm{~K}$ (top) to $10 \mathrm{~K}$ (bottom). The positive magnetoresistance that is enhanced at low temperature suggests a shrinking of electronic impurity wave function under a magnetic field. ${ }^{24}$ However, because our current device geometry allows for potential mixing between Hall and longitudinal resistance, pinning the exact origin of this behavior will require multiterminal magnetotransport studies in the future.

We find that further decreasing the temperature from $1.4 \mathrm{~K}$ to $0.3 \mathrm{~K}$ (Fig. 2c; red solid line, left axis) decreased the four-probe conductance negligibly. VT measurements on three additional $\mathrm{Ni}_{3}(\mathrm{HITP})_{2}$ rod devices showed a similar weak, positive dependence on temperature (Supplementary Figure 2). In contrast, a four-probe VT measurement of a polycrystalline $\mathrm{Ni}_{3}(\mathrm{HITP})_{2}$ film device (Fig. 2d; black solid line, left axis) revealed a conductance that decreased by orders of magnitude over a smaller temperature range.

To understand the VT behavior for these single rod and polycrystalline devices, we plot $W(T)=d \ln G / d \ln T$ against $T$ in a logarithmic scale as shown in Fig. 2c and Fig. 2d (dashed lines, right axes). This Zabrodskii plot scheme ${ }^{25}$ has been used for doped organic polymers, ${ }^{26}$ quantum dot films, ${ }^{27}$ and other systems, ${ }^{28}$ where the negative slope of the Zabrodskii plot indicates localization behaviors with a reduced activation gap. We observe a positive slope for all single rod devices, indicating metallicity in the absence of the strong localization at low temperatures. A small reduced activation energy value that decreases as temperature decreases is consistent with a non-zero conductance value as $T$ goes to $0 \mathrm{~K}$ (Fig. 2c inset). For the polycrystalline film device, we observe a negative slope in the Zabrodskii plot, indicating a semiconducting nature, where the conductance approaches 0 as $T$ goes to $0 \mathrm{~K}$ (Fig. $2 \mathrm{~d}$ inset). Literature precedence 28,29 
and additional data (Supplementary Figures 3 and 4) are consistent with our interpretation of metallicity or degenerate doping in $\mathrm{Ni}_{3}(\mathrm{HITP})_{2}$ (see Supplementary Discussion).

a
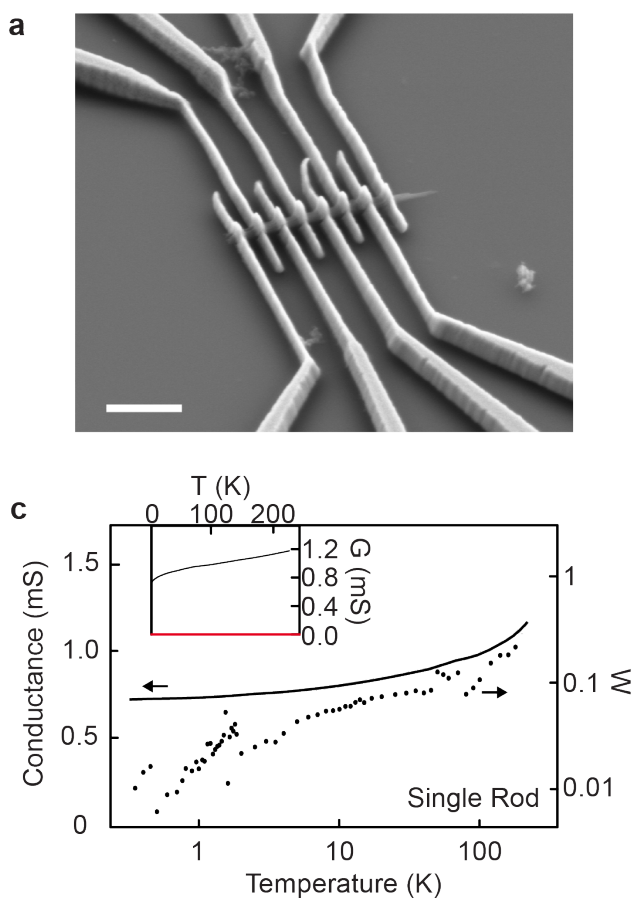
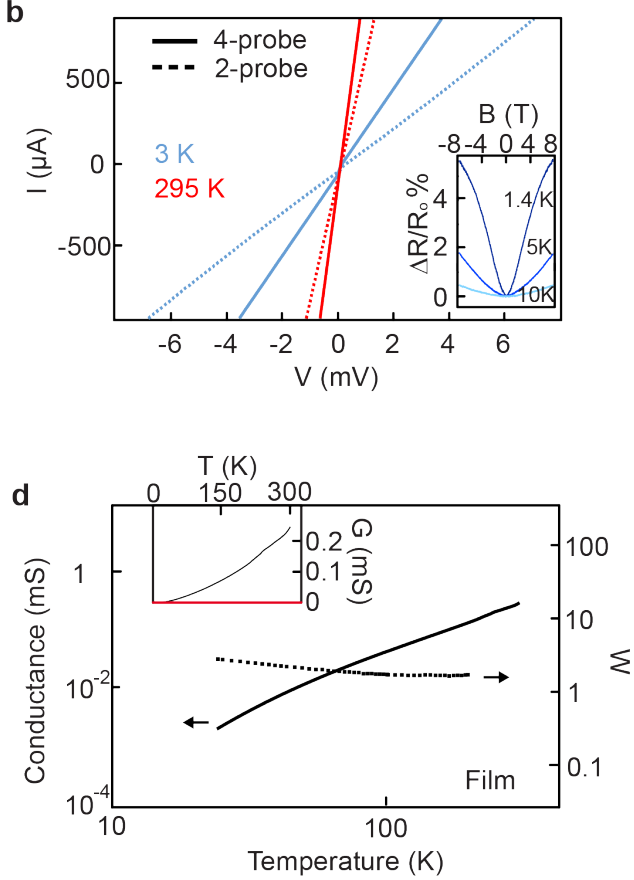

Figure 2 | Conductive 2D MOF devices of $\mathrm{Ni}_{3}(\mathrm{HITP})_{2}$. a, SEM of a single rod $\mathrm{Ni}_{3}(\mathrm{HITP})_{2}$ device with Ti/Pd contacts. Scale bar, $1 \mu \mathrm{m}$. b, Current-voltage plots of a single rod $\mathrm{Ni}_{3}(\mathrm{HITP})_{2}$ device at $295 \mathrm{~K}$ (blue) and $1.4 \mathrm{~K}$ (black). Inset: Normalized magnetoresistance at several fixed temperatures. c, $\mathbf{d}$ 4-probe temperature-dependent conductance (solid line; left axis) of a single rod device (c) and a polycrystalline film device (d) of $\mathrm{Ni}_{3}(\mathrm{HITP})_{2}$ and their corresponding Zabrodskii plots with their reduced activation energy (dotted lines; right axes). Inset: Temperature-dependent conductance plotted with linear axes.

We also isolated individual crystals of $\mathrm{Cu}_{3}(\mathrm{HHTP})_{2}$, and measured their conductivities as previous reports have indicated $\mathrm{Cu}_{3}(\mathrm{HHTP})_{2}$ to be isostructural to $\mathrm{Ni}_{3}(\mathrm{HITP})_{2}{ }^{14}$ For $\mathrm{Cu}_{3}(\mathrm{HHTP})_{2}$, thin nanocrystalline films ${ }^{14}$ or rods with diameters of $\sim 100 \mathrm{~nm}$ have been reported. ${ }^{19}$ Here, we obtain rods (Fig. 3a) or particles (Fig. 3b) of $\mathrm{Cu}_{3}(\mathrm{HHTP})_{2}$ with six-sided cross sections whose widths can be controlled synthetically (see Methods) from $100 \mathrm{~nm}$ up to 5 $\mu \mathrm{m}$ (Supplementary Figure 5). We also obtain thin flakes of $\mathrm{Cu}_{3}(\mathrm{HHTP})_{2}$ via techniques used to exfoliate traditional 2D materials. Sonication of washed $\mathrm{Cu}_{3}(\mathrm{HHTP})_{2}$ powder in isopropanol, 
water, or acetonitrile for $1-2 \mathrm{~h}$, followed by centrifugation and isolation of the suspended blue supernatant yields flakes with widths of $\sim 1 \mu \mathrm{m}$, and heights of $\sim 50-500 \mathrm{~nm}$ as evidenced by scanning electron microscope (SEM) and atomic force microscope (AFM) (Supplementary Figure 6).
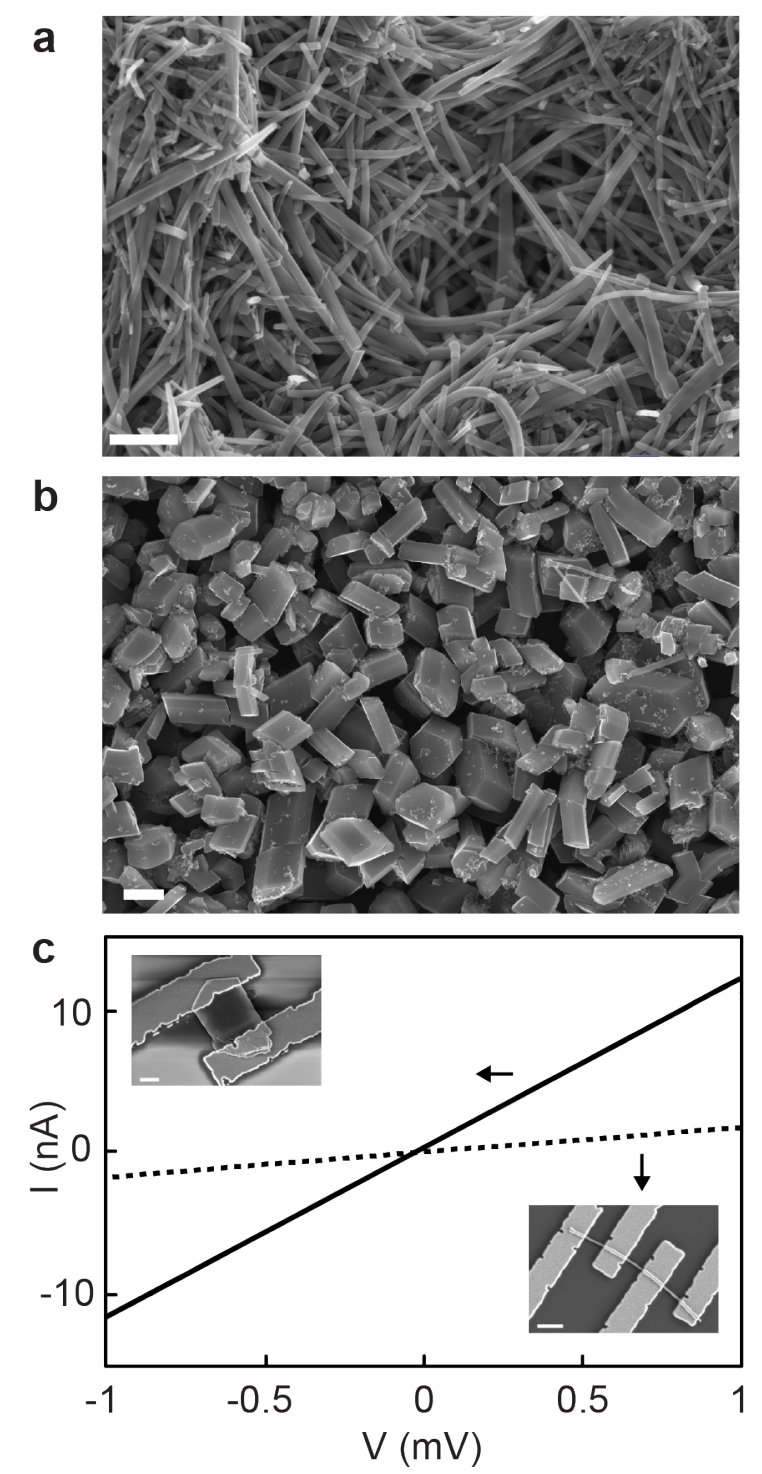

Figure 3 | $\mathbf{C u}_{3}$ (HHTP) $)_{2}$ a, b SEM images of $\mathrm{Cu}_{3}(\mathrm{HHTP})_{2}$ rods (a) and particles (b) obtained using distinct synthetic conditions (see Methods). Scale bars, $1 \mu \mathrm{m}$. Room temperature I-V curves of an exfoliated $\mathrm{Cu}_{3}(\mathrm{HHTP})_{2}$ flake (solid line) and $\mathrm{Cu}_{3}(\mathrm{HHTP})_{2}$ rod (dashed line) at 295 K. Insets show SEM images for corresponding devices. 
I-V curves for $\mathrm{Cu}_{3}(\mathrm{HHTP})_{2}$ rod and exfoliated flake devices (Fig. 3c), whose SEM images are shown as insets, yielded resistances of $562 \mathrm{k} \Omega$ and $84 \mathrm{k} \Omega$ at $295 \mathrm{~K}$, allowing us to estimate conductivities of $1.5 \mathrm{~S} / \mathrm{cm}$ and $0.5 \mathrm{~S} / \mathrm{cm}$, respectively, for these devices. Pellet conductivities of $0.1 \mathrm{~S} / \mathrm{cm}$ and $0.09 \mathrm{~S} / \mathrm{cm}$ were obtained from the $\mathrm{Cu}_{3}(\mathrm{HHTP})_{2}$ batch of particles used to exfoliate flakes, and the $\mathrm{Cu}_{3}(\mathrm{HHTP})_{2}$ rod batch, respectively. We note that we observe more variability in conductivity for $\mathrm{Cu}_{3}(\mathrm{HHTP})_{2}$ devices than for $\mathrm{Ni}_{3}(\mathrm{HITP})_{2}$ devices, which is likely related to difficulties with reliably forming ohmic contacts to $\mathrm{Cu}_{3}(\mathrm{HHTP})_{2}$, as indicated by the non-linear I-V curves observed for some devices as well as a significant decrease in conductance from $300 \mathrm{~K}$ to $200 \mathrm{~K}$, which prevents low temperature characterization. Despite the contact variability, over 10 single rod devices for $\mathrm{Cu}_{3}(\mathrm{HHTP})_{2}$ from different synthetic batches put a lower limit of conductivity at $\sim 0.1 \mathrm{~S} / \mathrm{cm}$ and indicate that in-plane and out-of-plane conductivities are of comparable orders of magnitude.

We performed high resolution (HR) TEM on individual crystals of $\mathrm{Ni}_{3}(\mathrm{HITP})_{2}$ and $\mathrm{Cu}_{3}(\mathrm{HHTP})_{2}$ to characterize their respective lattice structures, revealing key new insights into the stacking of 2D layers. These and other triphenylene-based MOFs have been reported to be nearly isostructural based on similarities in PXRD patterns, but the true nature of the stacking remained unclear. ${ }^{14}$ Imaging perpendicular to the long axis of a $\mathrm{Ni}_{3}(\mathrm{HITP})_{2}$ rod (Fig. 4a, left) reveals contrast fringes that run approximately parallel to the rod's axis. The fast Fourier transforms (FFTs) for this rod (Fig. 4a, middle) and others (Supplementary Figure 7) show the fringe periodicity to be $1.8 \mathrm{~nm}$ (red arrows), $0.9 \mathrm{~nm}$ (orange arrow), and $0.6 \mathrm{~nm}$ (blue arrow). Further inspection indicates an additional periodic feature (diffuse spot) with a spacing of $0.33 \mathrm{~nm}$ (white arrow) but with an orientation that is nearly perpendicular to the $1.8 \mathrm{~nm}$ family of fringes. The orthogonality of these spots in the FFT, combined with the periodic intensity profile (Fig. 4a, bottom) across the width of the rod, suggest that the $2 \mathrm{D}$ layers stack in an eclipsed or neareclipsed configuration, which we illustrate schematically in Fig. 4a, right. Specifically, the darker fringes (global minima in intensity profile) correspond to electron scattering along a higher metal and ligand density pathway, and the brighter fringes (local minima) to a lower density scattering pathway along the pore's center. The brightest fringes (global maxima) arise from scattering along vectors completely free of metal (Ni) atoms. PXRD and additional TEM data from other zone axis (Supplementary Figure 8), which show $1.1 \mathrm{~nm}$ spaced fringes and a hexagonal lattice, support this structural model (Fig. 4a). 

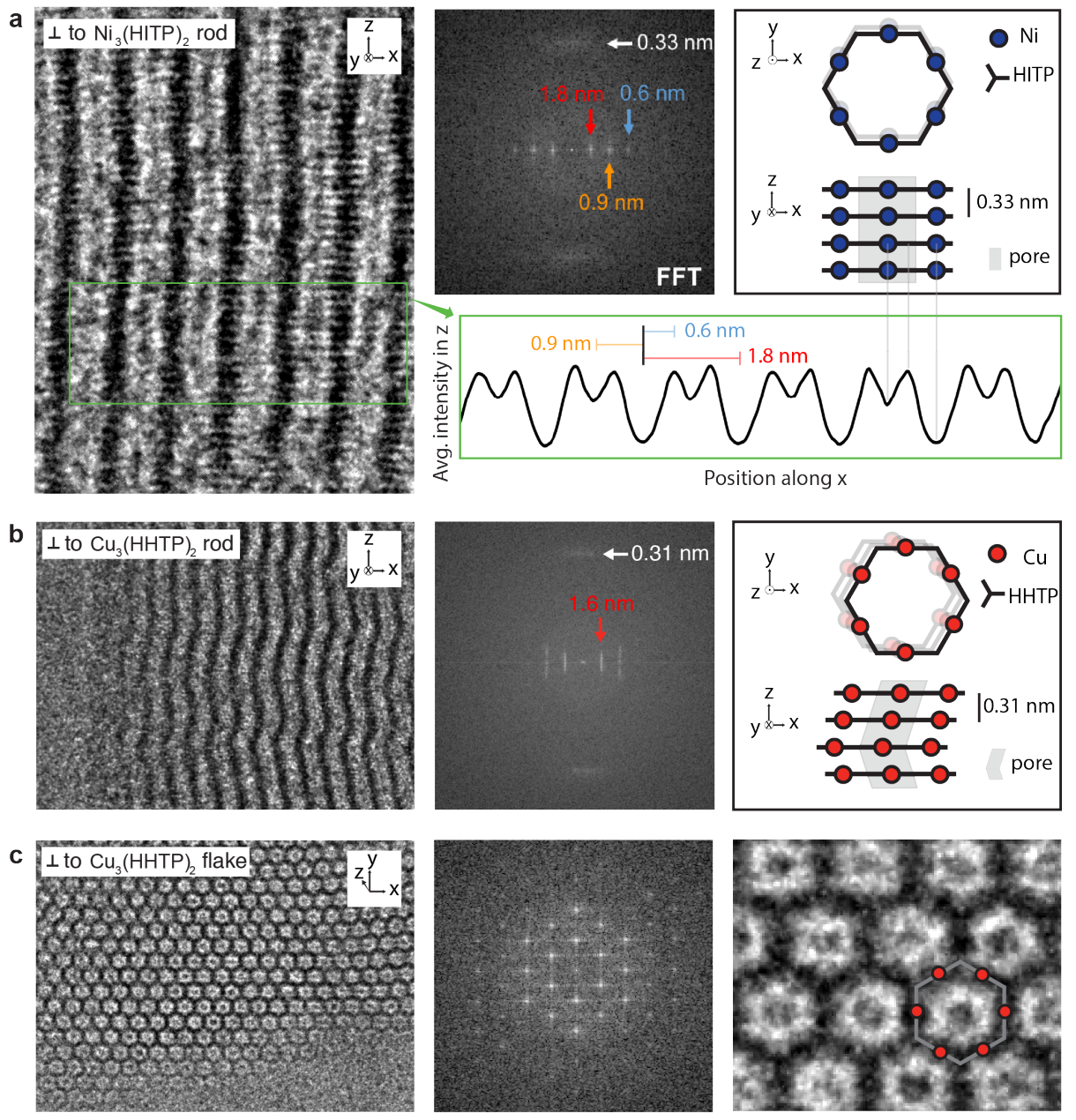

Figure 4 | TEM of $\mathrm{Ni}_{3}(\mathrm{HITP})_{2}$ and $\mathrm{Cu}_{3}(\mathrm{HHTP})_{2}$. a, Left: HRTEM image of a $\mathrm{Ni}_{3}(\mathrm{HITP})_{2} \operatorname{rod}$ whose long axis lies along the z-direction. Middle: FFT from a lower-magnification TEM image. Right: Schematic of $\mathrm{Ni}_{3}(\mathrm{HITP})_{2}$ structure illustrated for four layers in a near-eclipsed stacking configuration for imaging perpendicular (top schematic) and parallel (bottom schematic) to the 2D layers. Bottom: average intensity in the $\mathrm{z}$-direction measured along the $\mathrm{x}$-direction from the green box in the HRTEM image. The periodic features seen in the FFT are indicated with the red, orange, and blue lines. The grey lines extending from the intensity profile to the schematic indicate the pathways giving rise to the observed intensity profile. $\mathbf{b}$, Left: HRTEM image of a $\mathrm{Cu}_{3}(\mathrm{HHTP})_{2}$ rod whose long axis lies along the z-direction. Middle: FFT from a lowermagnification TEM image. Right: Schematic of the $\mathrm{Cu}_{3}(\mathrm{HHTP})_{2}$ structure illustrated for four layers in a near-eclipsed stacking configuration for imaging perpendicular (top schematic) and 
parallel (bottom schematic) to the 2D layers. The three top layers are depicted as stacked with an offset along the same direction while the bottom layer is stacked with an offset in the opposite direction, and represents a stacking fault. c, Low- (left) and high-magnification (right) HRTEM image of $\mathrm{Cu}_{3}(\mathrm{HHTP})_{2}$ flake obtained after exfoliation of $\mathrm{Cu}_{3}(\mathrm{HHTP})_{2}$ particles.

Surprisingly, HRTEM imaging perpendicular to the long axis of a $\mathrm{Cu}_{3}(\mathrm{HHTP})_{2}$ crystal, previously reported as isostructural with $\mathrm{Ni}_{3}(\mathrm{HITP})_{2},{ }^{14}$ and FFT analysis (Fig. 4b; Supplementary Figure 9) reveals a critical difference in the nature of stacking in $\mathrm{Cu}_{3}(\mathrm{HHTP})_{2}$ compared to that of $\mathrm{Ni}_{3}(\mathrm{HITP})_{2}$. That is, the eclipsed stacking model does not fit. For $\mathrm{Cu}_{3}(\mathrm{HHTP})_{2}$, the 2D layers are oriented horizontally and perpendicular to the rod axis (i.e. stacked from top to bottom in Fig. $4 \mathrm{~b}$, left), evidenced by the vertical position of the 0.31 spots in the FFT (white arrow). However, the angled orientation of the bright fringes in the HRTEM images indicate that the 2D layers are not oriented perpendicular to the pores axis. HRTEM and FFTs from other $\mathrm{Cu}_{3}(\mathrm{HHTP})_{2}$ rods (Supplementary Figure 10) confirm this finding, and suggest that the stacking orientation is tilted c.a. $15-23^{\circ}$ from a direction perpendicular to the pore axis. We propose that angled pores can arise by stacking subsequent layers with an offset along the same $a / b$ direction (Fig. 4b, right). Furthermore, the back-and-forth undulating nature of the pores observed in some rods (Fig. 4b, left) and the smearing of spots into lines in their respective FFTs (Fig. 4b, middle) can be explained by stacking faults or twin defects, as shown schematically with the bottom layer (Fig 4b, right and Supplementary Figure 9). Such defects would give rise to reversals in the stacking offset direction, and thus an undulating appearance. Additional data are consistent with this model where layers and pores are not perpendicular because of non-eclipsed stacking. First, SEM images of larger, faceted rods show six-sided cross sections that are not perpendicular to the rod's long axis, with clean cleavage planes that are similarly not normal to the rod's long axis (Supplementary Figure 11). Second, synchrotron PXRD data of $\mathrm{Cu}_{3}(\mathrm{HHTP})_{2}$ (Supplementary Figure 12) does not fit well with an eclipsed hexagonal unit cell but rather by a structure similar to the one outlined above (Fig. 4b, right). Third, TEM images (Fig. 4c, left) and FFTs (Fig. 4c, middle) of thin flakes obtained after exfoliation show a hexagonal crystalline structure with a honeycomb pattern that is consistent with imaging parallel to the pore axis $\left(\sim 20^{\circ}\right.$ from $z-$ direction). 
Further analysis and literature precedent suggest new insights into the 2D layer stacking that may be relevant to the growth, transport, and calculations thereof for 2D MOFs generally. The diffuse nature of the interlayer spots in the FFT for $\mathrm{Ni}_{3}(\mathrm{HITP})_{2}$ (white arrow; Fig. 4a, middle) and the range of pore angles for some CuHHTP rods (Fig. 4b, middle) are consistent with a variation in the stacking offset between layers, and may indicate that a strict assignment of AA or AB stacking with a single offset value, may not fully capture the structural features normal to the 2D MOF plane. Furthermore, the pores can stay continuous over hundreds of nanometers despite significant stacking variation over that length scale, indicating that the $2 \mathrm{D}$ layers are strongly ordered locally, where each subsequent layer is only offset by a small fraction of the 2D lattice's unit cell. This is consistent with previous calculations for $\mathrm{Ni}_{3}(\mathrm{HITP})_{2},{ }^{4}$ which predict a shallow potential energy surface for various stacking configurations that are near but not perfectly eclipsed (Supplementary Figures 7 and 9).

With the aid of single crystal device data, we make observations relevant to understanding transport in conductive 2D MOFs. First, for both $\mathrm{Cu}_{3}(\mathrm{HHTP})_{2}$ and $\mathrm{Ni}_{3}(\mathrm{HITP})_{2}$ systems, rod devices with significant out-of-plane contributions show conductivities greater than their polycrystalline pellet measurements. The out-of-plane transport is often disregarded in the literature compared to in-plane transport, but is consistent with band structure and density of states calculations, which show that $\mathrm{C}, \mathrm{N}$, and Ni contribute to these out-of-plane bands. Second, Zabrodskii plots show a positive slope for single crystal $\mathrm{Ni}_{3}(\mathrm{HITP})_{2}$ devices but a negative slope for its polycrystalline film, indicating intrinsic metallic nature for single crystal MOFs, with the observed non-metallic behavior of polycrystalline film likely extrinsic to the materials. Additional work is needed to further understand the role that anisotropy and grain boundaries contribute to polycrystalline transport. Importantly, for organic systems and quantum dot films, by controlling film preparation and the material's surface, conductance can be increased by orders of magnitude; similar strategies may yield significant increases in polycrystalline MOF transport. Last, we note that literature reports have attempted to claim metallicity or a semiconducting nature for various MOFs or coordination polymers by analyzing high temperature polycrystalline pellets or by applying Arrhenius equations to weak temperature dependences, which may lead to incorrect interpretations of small band gaps. We show that 
although the out-of-plane conduction for $\mathrm{Ni}_{3}(\mathrm{HITP})_{2}$ devices decreases with temperature, the Zabrodskii analysis at low temperatures and single crystals are critical in determining the nature of its conduction. 


\section{References}

1. Stassen, I. et al. An updated roadmap for the integration of metal-organic frameworks with electronic devices and chemical sensors. Chem. Soc. Rev. 46, 3185-3241 (2017).

2. Sun, L., Campbell, M. G. \& Dincă, M. Electrically Conductive Porous Metal-Organic Frameworks. Angew. Chem. Int. Ed. 55, 3566 (2016).

3. Ko, M., Mendecki, L. \& Mirica, K. Conductive two-dimensional metal-organic frameworks as multifunctional materials. Chem. Commun. 54, 7873-7891 (2018).

4. Sheberla, D. et al. High Electrical Conductivity in $\mathrm{Ni}_{3}(2,3,6,7,10,11$ hexaiminotriphenylene), a Semiconducting Metal-Organic Graphene Analogue. J. Am. Chem. Soc. 136, 8859-8862 (2014).

5. Dou, J.-H. et al. Signature of metallic behavior in the metal-organic frameworks $\mathrm{M}_{3}$ (hexaiminobenzene)2. J. Am. Chem. Soc. 139, 13608-13611 (2017).

6. Campbell, M. G. et al. $\mathrm{Cu}_{3}$ (hexaiminotriphenylene) 2 : an electrically conductive 2D metalorganic framework for chemiresistive sensing. J. Am. Chem. Soc. 137, 13780 (2015).

7. Dong, L. et al., Two-Dimensional $\pi$-Conjugated Covalent-Organic Frameworks as Quantum Anomalous Hall Topological Insulators. Phys. Rev. Lett. 116, 096601 (2016).

8. Jeon, I.-R. et al., A 2D Semiquinone Radical-Containing Microporous Magnet with SolventInduced Switching from Tc $=26$ to 80 K. J. Am. Chem. Soc. 137, 15699-15702 (2015)

9. Sheberla, D. et al. Conductive MOF electrodes for stable supercapacitors with high areal capacitance. Nature Mater. 16, 220-224 (2017).

10. Feng, D. et al. Robust and conductive two-dimensional metal-organic frameworks with exceptionally high volumetric and areal capacitance. Nature Energy 3, 30-36 (2018).

11. Miner, E. M. et al. Electrochemical oxygen reduction catalysedisêpiby $\mathrm{Ni}_{3}$ (hexaiminotriphenylene)2. Nature Comm. 7, 10942 (2016).

12. Clough, A. J., Yoo, J. W., Mecklenburg, M. H. \& Marinescu, S. C. Two-Dimensional Metal-Organic Surfaces for Efficient Hydrogen Evolution from Water. J. Am. Chem. Soc. 137, 118-121 (2015).

13. Campbell, M. G., Liu, S. F., Swager, T. M. \& Dincă, M. Chemiresistive Sensor Arrays from Conductive 2D Metal-Organic Frameworks. J. Am. Chem. Soc. 137, 13780-13783 (2015).

14. Rubio-Giménez, V. et al. Bottom-Up Fabrication of Semiconductive Metal-Organic Framework Ultrathin Films. Adv. Mater. 30, 1704291 (2018). 
15. Furukawa, H. et al. The Chemistry and Applications of Metal-Organic Frameworks. Science 341, 1230444 (2013).

16. Coudert, F.-X. Responsive Metal-Organic Frameworks and Framework Materials: Under Pressure, Taking the Heat, in the Spotlight, with Friends. Chem. Mater. 27, 1905-1916 (2015).

17. Huang, X. et al., Superconductivity in a Copper(II)-Based Coordination Polymer with Perfect Kagome Structure. Angew. Chem. Int. Ed. 57, 146-150 (2018).

18. Li, W. et al. Chemically diverse and multifunctional hybrid organic-inorganic perovskites. Nature Rev. Mater. 2, 16099 (2017).

19. Hmadeh, M. et al. New Porous Crystals of Extended Metal-Catecholates. Chem. Mater. 24, 3511-3513 (2012).

20. Kambe, T. et al. Redox Control and High Conductivity of Nickel Bis(dithiolene) Complex $\pi^{-}$ Nanosheet: A Potential Organic Two-Dimensional Topological Insulator. J. Am. Chem. Soc. 136, 14357-14360 (2014).

21. Aubrey, M. et al. Electron delocalization and charge mobility as a function of reduction in a metal-organic framework. Nature Mater. 17, 625-632 (2018).

22. Xie, L. S. et al. Tunable Mixed-Valence Doping toward Record Electrical Conductivity in a Three-Dimensional Metal-Organic Framework. J. Am. Chem. Soc. 140, 7411-7414 (2018).

23. Foster, M. E. et al. Unraveling the Semiconducting/Metallic Discrepancy in $\mathrm{Ni}_{3}(\mathrm{HITP})_{2}$. J. Phys. Chem. Lett. 9, 481-486 (2018).

24. Nguen, V. L., Spivak, B. Z. \& Shklovskii B. I. Tunnel hops in disordered systems. Sov. Phys.-JETP 62, 1021 (1985).

25. Zabrodskii, A. G. \& Shlimak I. S., Sov. Phys. Semicond. 9, 391 (1975).

26. Le, T.-H., Kim, Y. \& Yoon, H. Electrical and Electrochemical Properties of Conducting Polymers. Polymers 9, 150 (2017).

27. Liu, H., Pourret, A. \& Guyot-Sionnest, P. Mott and Efros-Shklovskii Variable Range Hopping in CdSe Quantum Dots Films. ACS Nano 4, 5211-5216 (2010).

28. Ahlskog, M., Reghu, M. \& Heeger, A. J. The temperature dependence of the conductivity in the critical regime of the metal-insulator transition in conducting polymers. J. Phys.:

Condens. Matter 9, 4145-4156 (1997). 
29. Kaiser, A. B. Electronic transport properties of conducting polymers and carbon nanotubes. Rep. Prog. Phys. 64, 1-49 (2001). 